\title{
Synthesis of Diaryl Disulfides, Sulfides and Aryl Alkyl Sulfides from Isothiazolones
}

\author{
BHAGAT SINGH BHAKUNI ${ }^{* 1}$, RAVINDER KUMAR ${ }^{2}$ and \\ PRAKASH SINGH BHAKUNI ${ }^{3}$
}

${ }^{1}$ Department of Chemistry, Kirori Mal College, University of Delhi, India

${ }^{2}$ Department of Chemistry, Sri Venkateshwara College, University of Delhi, India

${ }^{3}$ Echelon Institute of Technology, Faridabad, India

bhakunidu@gmail.com

Received 24 December 2015 / Accepted 8 January 2016

\begin{abstract}
Diaryl disulfides, sulfides and aryl alkyl sulfides were synthesized from isothiazolones. This method expedient and high yielding procedure for diaryl disulfides, sulfides and aryl alkyl sulfides under different reaction conditions. The structure of the compounds was determined with NMR, Mass Spectrometry and Infrared Spectroscopy.
\end{abstract}

Keywords: Isothiazolones, Diaryl Disulfides, Unsymmetrical Sulfides.

\section{Introduction}

Transition metal such as $\mathrm{Ni}, \mathrm{Cu}, \mathrm{Fe}$ etc. catalyzed carbon hetero-atom coupling reactions have used as a new tool to synthesize a different series of hetero-atom ( $\mathrm{S}, \mathrm{Se}$ and $\mathrm{Te}$ ) containing organic molecules ${ }^{1}$. Copper catalyzed synthesis of unsymmetrical diaryl sulfides, diaryl disulfides, arylthiols, arylsulfinamides and copper catalyzed 1,2-hydroxysulfenylation of alkenes has been well established ${ }^{2,3}$. Recently, catalytic methods to synthesize sulfur-nitrogen heterocycles (isothiazolones) have been reported by our group ${ }^{4}$. Mono sulphides and disulphides are importantnt organic moieties found in various biologically active molecules.These molecules had direct application to pharmateutical and agrochemicals. Grignard reaction ${ }^{5}$ is one of the easiest procedures to synthesis monosulphides from sulphur nitrogen heterocycles. Sulphur nitrogen hetero cycles can be converted to their respective disulphides simply reducing them by sodium borohydride.

\section{Experimental}

Synthesis of Bis(N-phenylbenzamide)disulfide(1)

2-Phenylbenzo[d]isothiazol-3(2H)-one (0.68 g, $3.0 \mathrm{mmol})$ was dissolved in EtOH (20 mL) and cooled to $0{ }^{\circ} \mathrm{C}$ under $\mathrm{N}_{2}$. To this solution, $\mathrm{NaBH}_{4}(0.19 \mathrm{~g}, 5.0 \mathrm{mmol})$ was added portion wise. Resulted colorless solution stirred for $30 \mathrm{~min}$ at this temperature and then room 
temperature for $45 \mathrm{~min}$. After this, reaction mixture was poured into a beaker containing crushed ice $(40 \mathrm{~g})$ and kept in air for oxidation for $3 \mathrm{~h}$. Standard workup and crystallization from $\mathrm{CH}_{2} \mathrm{Cl}_{2}$ afforded pale yellow crystalline solid. Yield $0.50 \mathrm{~g}(73 \%)$, mp $240-242{ }^{\circ} \mathrm{C}$ $\left(245-247{ }^{\circ} \mathrm{C}\right)^{6} .{ }^{1} \mathrm{H}$ NMR $\delta 10.6(\mathrm{~s}, 2 \mathrm{H}), 7.78-7.72(\mathrm{~m}, 6 \mathrm{H}), 7.63(\mathrm{~d}, \mathrm{~J}=8.0 \mathrm{~Hz}, 2 \mathrm{H}), 7.54-$ $7.49(\mathrm{~m}, 2 \mathrm{H}), 7.42-7.33(\mathrm{~m}, 6 \mathrm{H}), 7.16-7.09(\mathrm{~m}, 2 \mathrm{H}) .{ }^{13} \mathrm{C}$ NMR $\delta 170.9,144.1,141.7,139.9$, 136.6, 134.0, 133.7, 131.6, 131.5, 129.2, 125.4. ES-MS(ESI) $457.0\left(\mathrm{M}+\mathrm{H}^{+}\right)$. IR (plate): $3283,2924,1636,1599,1528,1430,1322,1256,1116 \mathrm{~cm}^{-1}$.

\section{Bis(N-cyclohexylbenzamide) Disulfide (2)}

Disulphide 2 was prepared from 2-cyclohexylbenzo[d]isothiazol-3(2H)-one using the same procedure used for the compound 1. Yield $0.74 \mathrm{~g}(90 \%),\left(249{ }^{\circ} \mathrm{C}\right)^{7} .{ }^{1} \mathrm{H}$ NMR $\delta 8.25(\mathrm{~d}, \mathrm{~J}=8.0$ $\mathrm{Hz}, 2 \mathrm{H}), 7.45$ (dd, J = 8.0, $1.5 \mathrm{~Hz}, 2 \mathrm{H}), 7.40(\mathrm{dd}, \mathrm{J}=8.0,1.0 \mathrm{~Hz}, 2 \mathrm{H}), 7.28$ (dt, J =8.0, $1.5 \mathrm{~Hz}$, $1 \mathrm{H}), 7.16(\mathrm{dt}, \mathrm{J}=7.5,1.2 \mathrm{~Hz}, 1 \mathrm{H}), 5.32(\mathrm{bs}, 2 \mathrm{H}), 3.71(\mathrm{~m}, 2 \mathrm{H}), 1.84(\mathrm{~m}, 4 \mathrm{H}), 1.73(\mathrm{~m}, 4 \mathrm{H}), 1.60$ $(\mathrm{m}, 2 \mathrm{H}), 1.36-1.26(\mathrm{~m}, 8 \mathrm{H}), 1.13(\mathrm{~m}, 2 \mathrm{H})\left({ }^{1} \mathrm{H}\right.$ NMR spectrum shows minor impurities at 8.43, 7.61 and $7.10 \mathrm{ppm}$ and could be due to S-N heterocycle ). ${ }^{13} \mathrm{C}$ NMR $\delta 167.3,134.3,133.4,130.6$, 130.4, 128.6, 124.9, 48.7, 32.7, 25.7, 25.2. ES-MS(ESI) 469.1 $\left(\mathrm{M}+\mathrm{H}^{+}\right)$

\section{2-(Methylthio)-N-phenylbenzamide (3)}

To the reduced reaction mixture of 2-phenylbenzo[d]isothiazol-3(2H)-one (at $3.0 \mathrm{mmol}$ scale as described for the synthesis of compound 1), methyl iodide $(0.3 \mathrm{~mL}, 5.0 \mathrm{mmol})$ was added at $0{ }^{\circ} \mathrm{C}$ and resulted reaction mixture was stirred for $30 \mathrm{~min}$. Standard workup and purification by column chromatography on silica gel using hexane/ethyl acetate (8:2) yielded 3 as white crystalline solid. Yield $0.67 \mathrm{~g}(92 \%), \mathrm{mp} 150-152{ }^{\circ} \mathrm{C}\left(148-149{ }^{\circ} \mathrm{C}\right)^{6} .{ }^{1} \mathrm{H}$ NMR $\delta 8.37(\mathrm{~s}, 1 \mathrm{H}), 7.74(\mathrm{~d}, \mathrm{~J}=8.0 \mathrm{~Hz}, 1 \mathrm{H}), 7.68(\mathrm{~d}, \mathrm{~J}=8.0 \mathrm{~Hz}, 2 \mathrm{H}), 7.45(\mathrm{t}, \mathrm{J}=7.5 \mathrm{~Hz}$, $2 \mathrm{H}), 7.28(\mathrm{t}, \mathrm{J}=7.5 \mathrm{~Hz}, 1 \mathrm{H}), 7.19(\mathrm{t}, \mathrm{J}=8.0 \mathrm{~Hz}, 2 \mathrm{H}), 7.17(\mathrm{t}, \mathrm{J}=7.5 \mathrm{~Hz}, 1 \mathrm{H}), 2.51(\mathrm{~s}, 3 \mathrm{H})$. ${ }^{13} \mathrm{C}$ NMR $\delta 166.0,137.9,136.6,135.3,131.1,129.16,129.09,128.1,125.8,124.6,120.0$, 17.1. IR (plate): $3298,2923,1646,1601,1542,1436,1325,1252,1164 \mathrm{~cm}^{-1}$. HRMS (ESI) $m / z 266.0647$ calculated for $\mathrm{C}_{14} \mathrm{H}_{13} \mathrm{NOS}+\mathrm{Na}^{+} 266.0612$.

$N$-Benzyl-2-(phenylthio)benzamide (4) ${ }^{7}$

2-Benzylbenzo[d]isothiazol-3(2H)-one $(0.79 \mathrm{~g}, 3.5 \mathrm{mmol})$ was dissolved in dry THF $(15 \mathrm{~mL})$. Phenylmagnesium bromide was synthesized in THF $(25 \mathrm{~mL})$ from bromobenzene $(0.75 \mathrm{~mL}, 4.0 \mathrm{mmol})$ and $(187 \mathrm{mg}, 4.8 \mathrm{mmol})$ on $4.0 \mathrm{mmol} \mathrm{scale}$. To the Grignard reagent, 2-benzylbenzo[d]isothiazol-3(2H)-one was added drop wise via syringe at $60{ }^{\circ} \mathrm{C}$. After complete addition, resulted milky colored solution was obtained which was heated at $60{ }^{\circ} \mathrm{C}$ for an additional hour and then poured over crushed ice $(50 \mathrm{~g})$. Standard workup and purification by column chromatography using hexane / ethyl acetate (7.5: 2.5) gave white solid. Yield $0.89 \mathrm{~g}(80 \%), \mathrm{mp} 110-112{ }^{\circ} \mathrm{C} .{ }^{1} \mathrm{H}$ NMR $87.71-7.69(\mathrm{~m}, 1 \mathrm{H}), 7.34-7.22$ (m, $13 \mathrm{H}), 6.91-6.75(\mathrm{~m}, 1 \mathrm{H}), 4.61(\mathrm{~d}, \mathrm{~J}=4.0 \mathrm{~Hz}, 2 \mathrm{H}) .{ }^{13} \mathrm{C} \mathrm{NMR} \delta 167.6,137.8,136.5,134.6$, $134.3,132.3,131.7,130.9,1 \mathrm{~S} 29.5,129.1,128.7,128.0,127.7,127.5,127.1,44.2$. IR (plate): 3298, 3061, 2923, 1643, 1531, 1436, 1306, 1263, 1157, $1024 \mathrm{~cm}^{-1}$. HRMS (ESI) $\mathrm{m} / z$ 342.0929 calculated for $\mathrm{C}_{20} \mathrm{H}_{17} \mathrm{NOS}+\mathrm{Na}+342.0944$.

\section{$N$-(4-Methoxyphenyl)-2-(phenylthio)benzamide (5)}

Diaryl sulfide 5 was prepared from 2-cyclohexylbenzo[d]isothiazol-3(2H)-one by following similar procedure as described for compound 4 at $4 \mathrm{mmol}$ scale. Purification by column chromatography using hexane / ethyl acetate (8: 2) yielded a white crystalline solid. Yield $1.17 \mathrm{~g},(87 \%), \mathrm{mp} 142-144{ }^{\circ} \mathrm{C} .{ }^{1} \mathrm{H}$ NMR $\delta 8.25(\mathrm{~s}, 1 \mathrm{H}), 7.76(\mathrm{~d}, \mathrm{~J}=8.0 \mathrm{~Hz}, 1 \mathrm{H}), 7.46(\mathrm{~d}, \mathrm{~J}=$ $8.0 \mathrm{~Hz}, 2 \mathrm{H}), 7.37-7.29(\mathrm{~m}, 9 \mathrm{H}), 6.87(\mathrm{~d}, \mathrm{~J}=8.0 \mathrm{~Hz}, 1 \mathrm{H}), 3.81(\mathrm{~s}, 3 \mathrm{H}) .{ }^{13} \mathrm{C}$ NMR $\delta$ 165.6, 
$156.6,136.9,134.52,134.27,132.4,131.7,131.03,130.95,129.54,129.41,127.8,127.4$, 121.9, 114.1, 55.6. IR (plate): 3287, 2924, 2852, 1643, 1598, 1513, 1410, 1247, 1175, $1027 \mathrm{~cm}^{-1}$. HRMS (ESI) $\mathrm{m} / \mathrm{z} 336.1044$ and 358.0867 (calcd for $\mathrm{C}_{20} \mathrm{H}_{17} \mathrm{NO}_{2} \mathrm{~S}+\mathrm{H}^{+}: 336.1053$ and $\mathrm{C}_{20} \mathrm{H}_{17} \mathrm{NOS}+\mathrm{Na}^{+}: 358.0872$ ).

\section{Instrumentations}

All NMR experiments were carried out on 400 or $500 \mathrm{MHz}$ spectrometers in DMSO-d $\mathrm{d}^{6}$ or $\mathrm{CDCl}_{3}$ and NMR chemical shifts are reported in ppm referenced to the solvent peaks of $\mathrm{CDCl}_{3}$ $7.26 \mathrm{ppm}$ for ${ }^{1} \mathrm{H}$ and $77.0( \pm 0.1) \mathrm{ppm}$ for ${ }^{13} \mathrm{C}$, respectively) or DMSO-d ${ }^{6}(2.50 \mathrm{ppm}$ for $1 \mathrm{H}$ and $39.50 \mathrm{ppm}$ for $13 \mathrm{C}$, respectively). Mass analysis was performed on quadruple-time offlight (Q-TOF) mass spectrometer equipped with an ESI source (+ve). Infrared spectra were recorded as a pellet in $\mathrm{KBr}$ with a FTIR machine. Melting points are uncorrected. DMF with sure seal septa, sulphur powder, copper iodide and 1,10-phenanthroline were used as received from Aldrich. Grinded anhydrous $\mathrm{K}_{2} \mathrm{CO}_{3}$ powder was used which was grinded using mortar, dried in oven at $160{ }^{\circ} \mathrm{C}$ for $6 \mathrm{~h}$ and stored in a desiccator.

\section{Results and Discussion}

To explore the synthetic utility of synthesized isothiazolones, we were able to synthesize corresponding disulfides, unsymmetrical diaryl and aryl alkyl sulfides from synthesized S-N heterocycles (Scheme 1). Diaryl disulfides (1-2) and aryl alkyl sulfide (3) was obtained by the reduction of $\mathrm{S}-\mathrm{N}$ bond using $\mathrm{NaBH}_{4}$.

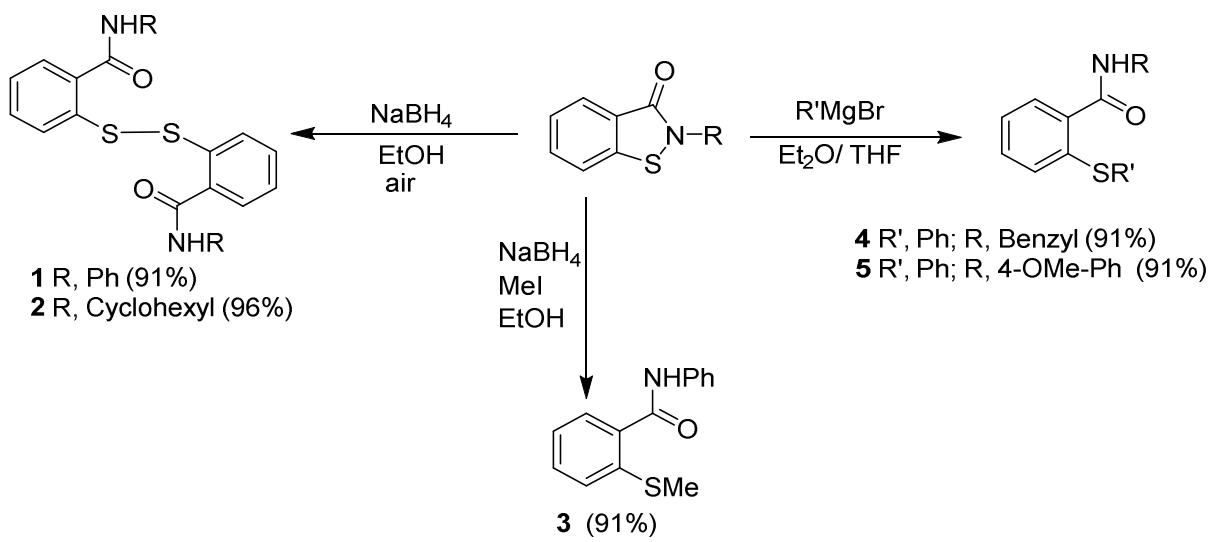

Scheme 1. Synthesis of Diaryl disulfides, -Sulfides and Aryl alkyl sulfides

Aerial oxidation of reduced reaction mixture of gave respective disulfides $\mathbf{1}$ and $\mathbf{2}$, respectively. Addition of methyl iodide to the reduced reaction mixture of 2-phenylbenzo [d]isothiazol-3(2H)-one gave methylsulfide 3 in one pot. Quantitative conversion of S-N heterocycles into unsymmetrical diaryl sulfides $\mathbf{4}$ and $\mathbf{5}$ has been optimized by employing 1.5 equiv. of Grignard's reagent.

\section{Acknowledgment}

We thanks, Department of Chemistry, IIT Bombay for mass and HRMS data collections.

\section{References}

1. Beletskaya I P and Ananikov V P, Chem Rev., 2011, 111(3), 1596-1636; DOI:10.1021/cr100347k 
2. (a) Taniguchi N, J Org Chem., 2004, 69(20), 6904-6906; DOI:10.1021/jo040184q (b) Taniguchi $\mathrm{N}$ and Onami $\mathrm{T}, J$ Org Chem., 2004, 69(3), 915-920; DOI:10.1021/jo030300+ (c) Taniguchi N, Synlett, 2005, 1185-1187; DOI:10.1055/s2005-865230 (d) Taniguchi N, J Org Chem., 2006, 71(20), 7874-7876; DOI:10.1021/jo0608341 (e) Taniguchi N, J Org Chem., 2007, 72(4), 1241-1245; DOI:10.1021/jo062131+(f) Taniguchi N, Eur J Org Chem., 2010, 2010(14), 26702673; DOI:10.1002/ejoc.201000167

3. (a) Jiang Y, Qin Y, Xie S, Zhang X, Dong J, Ma D, Org Lett., 2009, 11(2), 52505253; DOI:10.1021/o1902186d (b) Ke F, Qu Y, Jiang Z, Li Z, Wu D and Zhou X, Org Lett., 2011, 13(3), 454-457; DOI:10.1021/ol102784c

4. (a)Bhakuni B S, Balkrishna S J, Kumar A and Kumar S, Tett Lett., 2012, 53(11), 1354-1357; DOI:10.1016/j.tetlet.2012.01.003 (b) Lisiak R and Młochowski J, Synth Commun., 2009, 39, 3141-3155; DOI:10.1080/00397910902730994

5. Domagala J M, Bader J P, Gogliotti R D, Sanchez J P, Stier M A, Song Y, Prasad J V N V, Tummino P J, Scholten J, et al., Bioorg Med Chem., 1997, 5(3), 569-579; DOI:10.1016/S0968-0896(96)00269-6

6. El-Barbary A A, Clausen K, Lawesson S O, Tetrahedron, 1980, 36(22), 3309-3315; DOI:10.1016/0040-4020(80)80182-7

7. Bao M, Shimizu M, Shimada S, Inoue J, Konakahara, Tetrahedron, 2004, 60(50), 11359-11366; DOI:10.1016/j.tet.2004.09.093 